\title{
PRIVATE NETWORK OPTIMIZATION
}

\author{
Aadil Gani Ganie \\ PhD Student, Institute of Information Sciences, University of Miskolc \\ 3515 Miskolc, Miskolc-Egyetemváros, e-mail: aadilganiganie@gmail.com
}

\begin{abstract}
The main challenging task in laying a network is not to define its topology but to calculate its costbenefit analysis, which is a research-oriented work. In any network, be it intra or internetwork, the main focus is always on optimizing cost and bandwidth. PSWAN is a medium-sized network at the Directorate of information technology India; this networking project provides internet services to many governmental and private offices. Optimization was required as the number of devices connected to this network was more. Two parameters that were optimized are 1. Bandwidth, and 2. Cost. Cost optimization was achieved by shifting some small networks like school or medium-size office to broadband. While bandwidth optimization was achieved by global load balancing, minimize latency, packet loss monitoring and bandwidth management. During optimization, no hardware change was done; Opnet software was used to calculate the results. The proposed optimized network architecture shows promising results as compared to the existing one.
\end{abstract}

Keywords: network protocols, network monitoring, optimization, bandwidth

\section{INTRODUCTION}

With increased dependency on the internet and advancements in cloud computing services, most of the application is delivered on the public internet. IT is becoming omnipresent in today's world [1]. Most of the services provided by enterprises and government institutions require the internet; this insistence or stipulation gives rise to network optimization tools and techniques. There are two ways of measuring network performance - passive and active. Passive measurement tools monitor applications already on the network, gathering performance data metrics. This gives a realistic representation of real-time conditions since it focuses on real apps. There is also no danger of network disruption, as there is no additional traffic. Active measurement tools generate data tailored to baseline performance. This does require other traffic, so it must be scheduled at appropriate times. The most important part of the network is planning data with equal priority, independent of the source and destination. Hence, there is no trivial technique to assure a specific transmission rate [2]. The two standard methods to measure the available bandwidth and latency are passive and active techniques [3].

Network performance metrics are network attributes that point to the health of a network. Network performance metrics like latency, packet loss, jitter, congestion and bandwidth availability are crucial to the end-user experience. For example, Network latency determines application-specific performance metrics like page load times, VoIP call quality, video buffering times and online gaming lag times. Packet loss leads to online games losing frames and jumping around-jitter results in cutoffs during VoIP calls [4]. Network optimization tools are reactive and require manual changes to network topology to affect performance. Network optimization has always been a core problem domain in operations research, as well as in computer science, applied mathematics, and many fields of engineering and 
management. The varied applications in these fields occur 'naturally' on some transparent physical network and in situations that are pretty unrelated to networks.

Moreover, because network optimization problems arise in so many diverse problem contexts, applications are scattered throughout the literature in several fields. Consequently, it is sometimes difficult for the research and practitioner community to fully appreciate the richness and variety of network applications [5]. The internetworking project PSWAN serves internet facilities to many persons, be it an organization or for personal use. It provides all the services from emailing to sharing multimedia and from browsing to social media. The load on the network increases with the increasing number of users added day by day. It has been observed that during the rush hours between $9 \mathrm{pm}$ to $11 \mathrm{pm}$, the internet speed decreases with the increase in the number of users, so network optimization was needed. After altering some of the existing network parameters using Opnet software, there was substantial bandwidth and speed. The proposed network architecture has optimized bandwidth and cost, as shown in this article's result section.

There is a difference between throughput and bandwidth; throughput tells you how much data was transferred from a source at any given time, and bandwidth means how much data could theoretically be transferred from a source at any given time. Knowing how both throughput and bandwidth perform is crucial for administrators to get a clear picture of the network's performance. As always, there's software available to make this process easier and quicker potentially. By far, the most important thing to do when optimizing throughput is to minimize network latency. Latency slows down throughput, which, in turn, lowers throughput and delivers poor network performance to users. Generally speaking, you want to minimize lag by monitoring endpoint usage and addressing network bottlenecks. The most common cause of latency is having too many people trying to use a network simultaneously. Latency gets even worse if multiple people are downloading simultaneously. In recent years bandwidth consumption has become an increasing concern for most organizations as bandwidth-intensive sites and video streaming has become more popular. However, understanding the current Internet environment is challenging due to the complexity of Internet applications. There is no simple way to understand the network's behaviours [6] truly. Various parameters contribute to network optimization and performance; these parameters, with their brief description, will be discussed in this section.

Choosing a suitable protocol for any network is very difficult. A routing protocol must not be configured if network complexity, multiple vendor devices and future expansion in the network are not thoroughly investigated. [6]. Different static and dynamic routing. Options are available [7]. The chief advantage of dynamic routing over static routing is scalability and adaptability. A dynamically routed network can grow more quickly and larger and adapt to changes in the network topology of this growth or the failure of one or more network components [4]. Simulation-based routing protocol performances have been analyzed by [8], which revealed the efficiency of EIGRP over RIP, which uses a single routing metric and exchanges periodic copies of the entire routing table, which makes the RIP network congested[9]. The capability of EIGRP to utilize bandwidth efficiently and its knowledge about the whole network using a topology table makes EIGRP an efficient routing option in an enterprise network $[10]$.

\section{RESULTS AND DISCUSSION}

There are various simulation tools; it has been observed that OPNET shows better accuracy and precision than other simulations tools. OPNET provides a comprehensive development environment for the specification, simulation and performance analysis of communication networks [6]. OPNET is 
extensive and powerful simulation software with a wide variety of capabilities. It enables the possibility to simulate entire heterogeneous networks with various protocols [4]. OPNET Modeler is a powerful tool that evaluates the web efficiently and accurately and predicts network behaviour before implementing it in a natural environment [10]. OPNET, with its unique approach, can provide an objective and reliable quantitative basis for network planning and design, and it could shorten the network construction period, improve the exactitude of decision making on network building and reduce the risk of network construction[11].

Network analysis, also known as traffic analysis, protocol analysis, sniffing, eavesdropping, and so on, captures network traffic and inspects it closely to determine what is happening on the network. A network analyzer decodes the data packets of standard protocols and displays the network traffic in a readable format. NetADHICT software has been used for network monitoring. We calculated both daily usage and weekly usage. We realized that network usage is much higher in the slots between $4 \mathrm{pm}$ and $6 \mathrm{pm}$ and again between $9 \mathrm{pm}$ and $11 \mathrm{pm}$. So, we got the busiest slots for which need the optimization. We continued to monitor the network until we reached satisfactory results, and the graph doesn't fluctuate much. The whole idea behind monitoring was identifying the busiest slot, which is causing the network to slow.

The existing network can provide internet services to more users than it already has by optimizing it. It has been observed that the current network has some flaws in assigning the bandwidth, and the new network protocol was introduced, which is more pertinent than the previous one. PSWAN is an operational network at the Directorate of information technology at Pondicherry, India. Almost 3000 systems are enjoying the internet services of PSWAN[12]. Since the bandwidth is the primary concern in any network, same was with this network, the number of users is more but the bandwidth required is significantly less, optimization of the network is necessary[13], to optimize the network, we pointed out some of the parameters that upon modification or change will provide better results and subsequently will optimize the network, these parameters are 1. Type of protocol 2. Type of topology 3. Access policies 4 . Traffic bottleneck 5. Bandwidth utilization and 6. Load balancing.

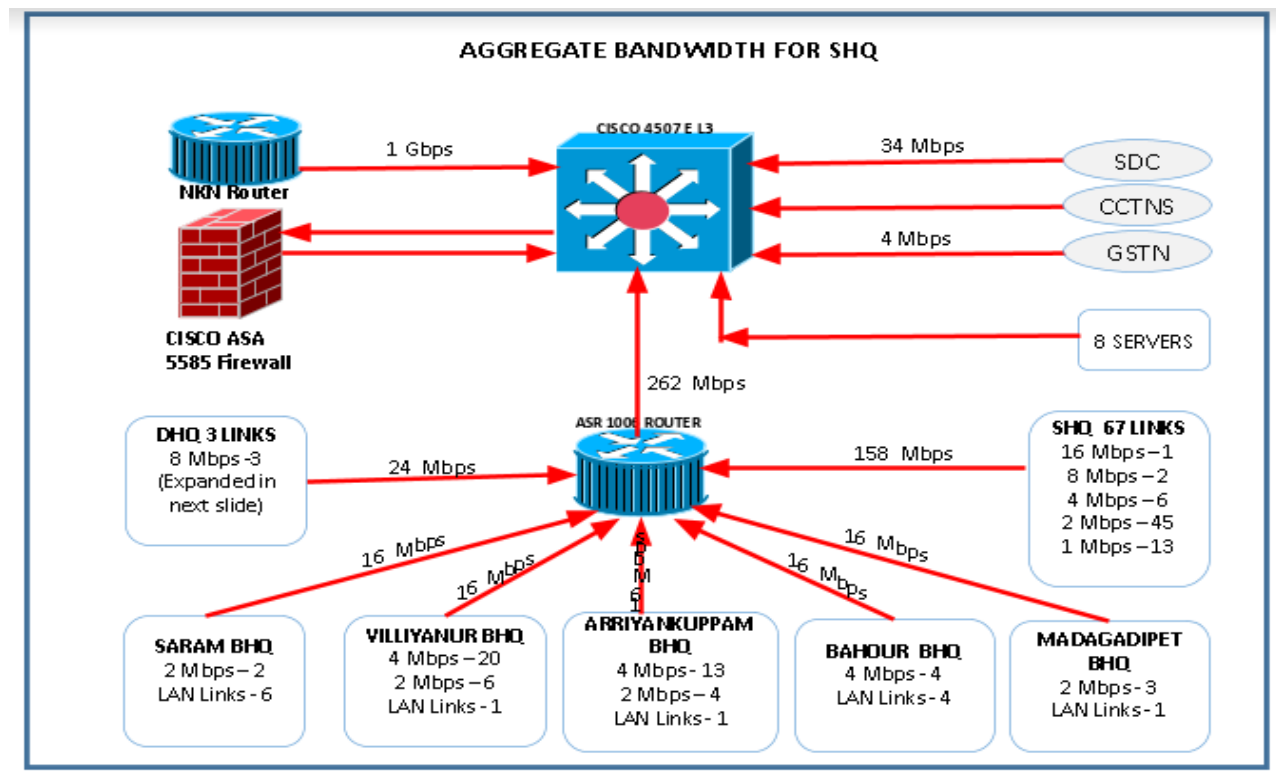

Figure 1. Existing network architecture. 
After modifying the parameters like 1. Type of protocol 2. Type of topology 3. Access policies 4 . Traffic bottleneck 5. Bandwidth utilization and 6. Load balancing the following results were obtained. PSWAN used OSPF (Open shortest path first), a generic protocol, and is device independent; however, it is a link-state protocol. However, there are specific issues with this protocol, such as its complexity and high CPU usage. Since most of the devices in PSWAN are Cisco devices, so EIGRP (Enhanced interior gateway routing protocol) suits it better as it a Cisco proprietary. Also, it uses a hybrid protocol, and it is simple compared to OSPF, and its CPU usage is also low. We can conclude from the above facts that OSPF is resource-intensive compared to EIGRP and gives us the edge to use EIGRP instead of existing OSPF. Upon simulation, EIGRP showed the better result, as shown in Figure 2. This comparison is based on network convergence duration between EIGRP and OSPF on the same network; it is evident that EIGRP's convergence rate is much higher than OSPF, which shows its effectiveness and power to optimize the network. A comparison was made between the two protocols based on network convergence duration and without a firewall, but results didn't change for EIGRP; it shows better results than OSPF.

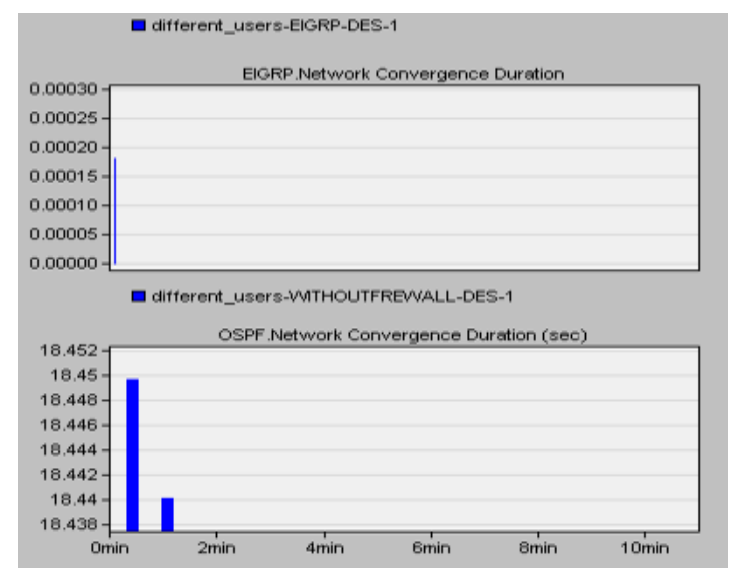

Figure 2. EIGRP vs OSPF.

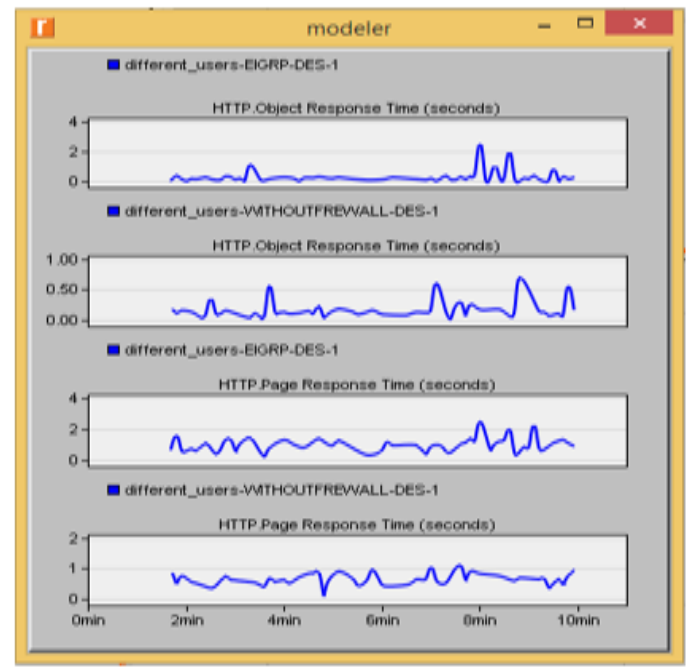

Figure 3. Object and page response between existing and proposed network. 
The second comparison was made between HTTP object response time between the existing network and the proposed network; the results showed that the proposed network's object response is significantly less than the existing network. Another graph based on HTTP page response time in seconds between the two networks was drawn, showing the proposed network in bright light. Using the Opnet software, both the networks were simulated using different parameters; upon execution, the proposed network model showed better results than the existing one. Below are the results with the images.

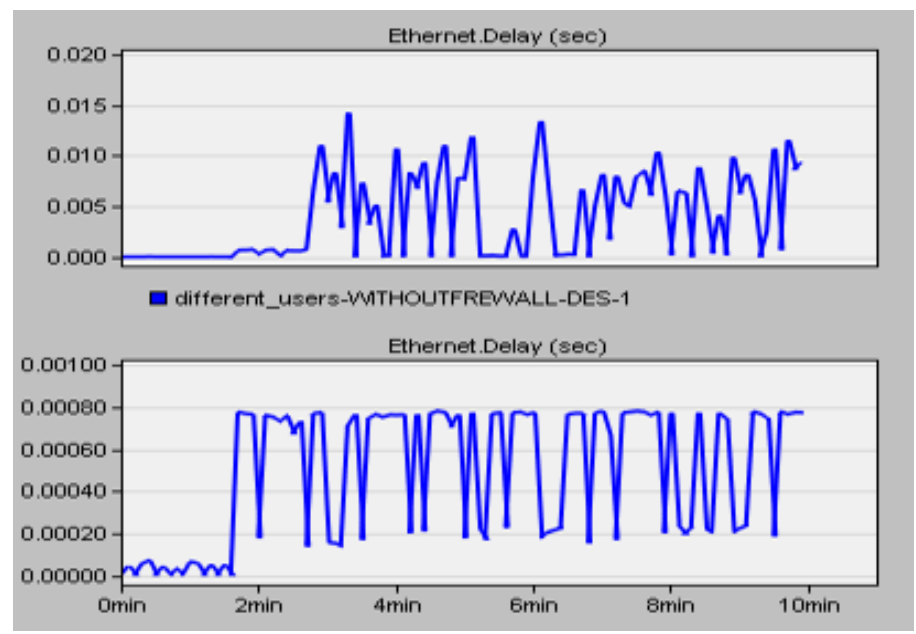

Figure 4. Ethernet delay comparison.

Ethernet delay is an important metric to measure the performance of any network. We compared the existing network with the proposed using Opnet software; the ethernet delay for the proposed network is significantly less. The highest ethernet delay in the current network is 0.0008 , while the ethernet delay for the existing network is much higher than 0.015 .

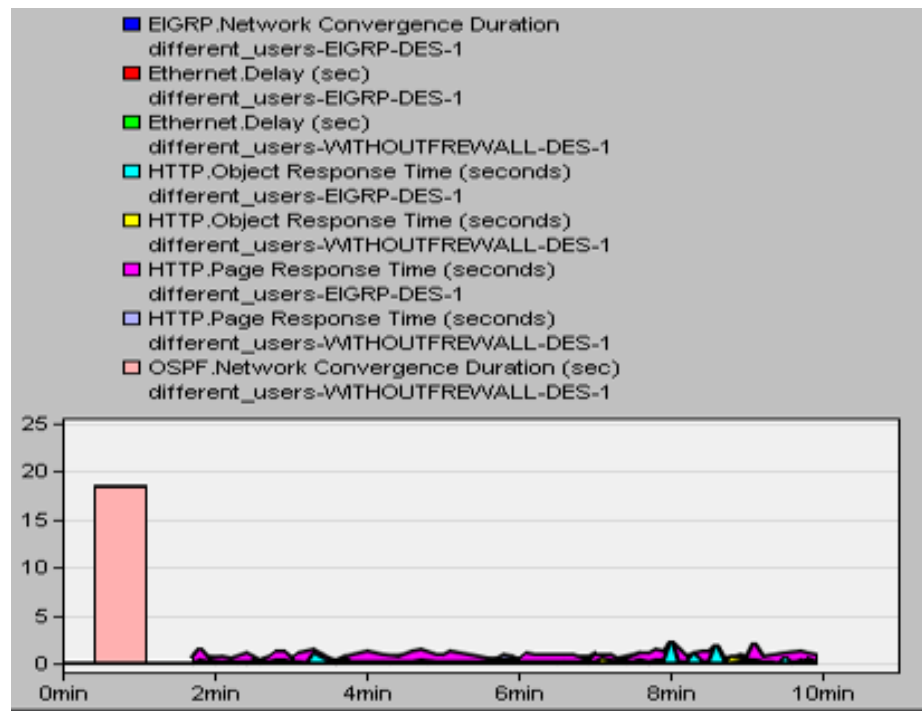

Figure 5. Combined comparison between existing and proposed network. 
It can be observed from the simulation that the proposed network is performing better than the existing network. If these parameters are changed in the physical network, it will show far better results and, consequently, lead to optimization.

\section{Conclusion}

In this paper, a case study of an existing operational network (PSWAN) has been considered. The previous network needs improvement to speed up the performance. Network performance has been increased substantially by modifying the network protocols and specific parameters. The first existing network was studied extensively to identify the weak points, and a new model has been proposed which overcomes the weak points in the existing network. Both the network models were analyzed using Opnet and Netadhict software. The parameters chosen showed promising results for the proposed network architectural model. More improvement can be made, and network can be optimized more with sophisticated devices and reprogramming the network protocols

\section{References}

[1] Busru, S.A., Ovies, P.M., Ganie, A.G. Enterprise Information Technology Governance Insights from India Listed Companies. Asian Journal of Information Technology 2018, 17(6):312-320. https://doi.org/10.36478/ajit.2018.312.320

[2] Deepika, M.S., Babu, K.N.R.M. An Approach to Effective Bandwidth Utilization using Software Define Networking. International Journal of Computer Science and Information Technologies 2014, 5(4): 5571-5574.

[3] Melander, B., Bjorkman, M., Gunningberg, P. Regression-Based Available Bandwidth Measurements. Proceedings of the 2002 International Symposium on Performance Evaluation of Computer and Telecommunications. pp.1-25.

[4] Thorenoor, S.G. Dynamic Routing Protocol Implementation Decision between EIGRP, OSPF and RIP Based on Technical Background Using OPNET Modeler. Proceedings of the 2010 Second International Conference on Computer and Network Technology, Bangkok, Thailand. pp. 191-195. https://doi.org/10.1109/ICCNT.2010.66

[5] Ahuja, R.K., Magnanti, T.L., Orlin, J.B., Reddy, M.R. Applications of network optimization. In: Handbooks in Operations Research and Management Science 1995 7: 1-83. https://doi.org/10.1016/S0927-0507(05)80118-5

[6] Kuo, C.F., Pang, A.C., Chan, S.K. Dynamic routing with security considerations. IEEE Transactions on Parallel and Distributed Systems 2009, 20(1):48-58. https://doi.org/10.1109/TPDS.2008.73

[7] Balasubramanian, M., Dua, S., Perkins, L.A., Kraft, D.H., Iyengar, S.S. Evidence combination for traffic adaptive routing. 18th International Conference on Systems Engineering, Las Vegas, NV, USA, 2005, pp. 355-362. https://doi.org/10.1109/ICSENG.2005.40

[8] Overview of TCP/IP - TCP/IP Network Administration, 3rd Edition [Book]. https://www.oreilly.com/library/view/tcpip-network-administration/0596002971/ch01.html (accessed Apr. 01, 2021)

[9] Inoue, H., Jansens, D., Hijazi, A., Somayaji, A. NetADHICT: A Tool for Understanding Network Traffic. Proceedings of the 21st Large Installation System Administration Conference, Dallas, TX: USENIX Association, November 11-16, 2007. pp. 39-47. 
[10] Oetiker, T. Rand, D. The Multi Router Traffic Grapher. Proceedings of the Twelfth Systems Administration Conference. Boston, Massachusetts, December 6-11, 1998. pp. 141-148.

[12] A. G. Ganie, "Optimization of PSWAN in terms of cost and bandwidth," International Journal of Information Techonology and Business 2018, 1(1): 42-47. https://doi.org/10.24246/ijiteb.112018.42-47

[13] Samad, D., Gani, G.A. Analyzing and predicting spear-phishing using machine learning methods. Multidiszciplináris https://doi.org/10.35925/j.multi.2020.4.30 